\title{
16. THE STARS WITH DOUBLE ENVELOPES
}

\author{
V. P. TSESEVICH \\ Astronomical Observatory, Odessa, U.S.S.R.
}

In modern astronomy we acquire fundamental knowledge concerning the dimensions and the constitution of the exterior layers of giant stars by the study of eclipsing systems. The red giants which form part of the systems of VV Cephei and $\zeta$ Aurigae have been studied thoroughly from the changes that the spectra of these systems undergo when the light of the bright eclipsed star passes through the envelope of the rarefied giant. At the present time it is clear that stars having extended envelopes are not rare. Thus, for example, F. I. Loukatskaya [1] (at the Principal Astronomical Observatory of the Academy of Sciences of the Ukrainian S.S.R.) has studied the eclipsing star AW Pegasi in different parts of the spectrum; she has shown that this star possesses a semi-transparent envelope that produces a considerable part of the eclipse. It turns out besides that certain spectral lines, observed in the spectrum of the bright star, come from the semi-transparent envelope of the less luminous companion.

Shulberg [2], of the University of Odessa, has worked out according to the Kosyrev-Chandrasekhar theory of the structure of extended atmospheres a general method for the determination of the elements of an eclipsing binary system that possesses envelopes; he has shown that there takes place in the envelope of the star $\mathrm{V}_{444}$ Cygni a strong absorption of the light of the eclipsing star, which confirms the existence of semitransparent stellar envelopes.

The more recent work of the author in collaboration with I. G. Jdanova [3] of the Principal Astronomical Observatory of the Academy of Sciences of the Ukrainian S.S.R. includes an explanation of a new method for discovering extended envelopes in giant stars, which might be applied to the study of physical variable stars. The author is of the opinion that researches pursued in that way will yield important results. As is known, the variables of the RV Tauri type are quasi-periodic over more or less long intervals. They maintain for a long time a rigorous periodicity which then suddenly interrupts itself. After a certain lapse of time, in the course of which the light varies irregularly, the regular variations re-establish themselves with a period approximating the initial period. 
The light curve also undergoes changes. It occasionally recalls the light curves of the stars of the $\beta$ Lyrae type, and sometimes those of the typical cepheids.

In addition, in a great number of stars of the RV Tauri type there have been observed long-period variations in the mean brightness. The study of this phenomenon has established that these slow variations are not an exception, but rather are the general rule.

The following are some characteristic values for stars of the RV Tauri type:

$\begin{array}{ccccc}\begin{array}{c}P_{1} \\ \text { Star }\end{array} & \begin{array}{c}P_{2} \\ \text { (days) }\end{array} & \begin{array}{c}A_{\mathrm{I}} \\ (\mathrm{mag} .)\end{array} & \begin{array}{c}A_{\mathrm{II}} \\ (\mathrm{km} . / \mathrm{sec})\end{array} \\ \text { DF Cygni } & 49 \cdot 8 & 780 \cdot 2 & - & - \\ \text { R Sagittae } & 70 \cdot 8 & \text { I I I 2 } & 0 \cdot 55 & 23 \\ \text { RV Tauri } & 78 \cdot 7 & 1224 & 1 \cdot 70 & 19 \\ \text { U Monocerotis } & 92 \cdot 3 & 2320 & 0 \cdot 30 & 40 \\ P_{1} \text { : Period of the rapid variation. } & \\ P_{2} \text { : Period of the slow variation. } \\ A_{\mathrm{I}} \text { : Amplitude of the slow variation of brightness. } \\ A_{\mathrm{II}} \text { : Amplitude of the slow variation of the radial velocity. }\end{array}$

The comparison of the light curves with those of colour index and radial velocity, deduced from the observations of Joy and of Sanford, have led us to the following conclusions:

First of all, the rapid variations of the light and of the spectral characteristics reveal the following properties, common to all the stars examined.

(I) A careful colorimetric study of AC Herculis in three spectral regions shows that its colour index undergoes great variations. The maximum variations are produced during minimum light. The variation of the colour index between the limits of $+0 \cdot \mathrm{I}$ and $+\mathrm{I} \cdot-$, magnitudes does not correspond to a change of spectral class from $\mathrm{F}_{\mathrm{I}}$ to $\mathrm{K}_{4}$. Considerable deviations from thermodynamic equilibrium are evident.

(2) The curve of variation of the colour index is similar in form to the light curve, if one takes for its maximum the minimum colour index, corresponding to the maximum of the colour temperature. At the same time, the curve of colour index precedes that of light by 0.12 period.

(3) The curve of variation of the radial velocities constructed from the metallic absorption lines is in phase with the light curve, as in the cepheids (if the negative values of the velocities are plotted in the positive sense on the ordinate axis).

(4) The curve of variation of the radial velocities constructed from the absorption lines of hydrogen is in phase with the curve of variation of the colour index, preceding the light curve and that of the metallic-line radial velocities by $0 \cdot 12$ period. 
(5) At the moment corresponding to the minimum value of the colour index, many of the stars studied showed emission lines of hydrogen.

All this indicates that the rapid variations of light burst forth in different layers of the extended photosphere of the star. The perturbation that leads a new cycle of variations begins at the base of the photosphere. The advance in phase of the curves of colour index and of hydrogen-line radial velocities, which has been discussed, speaks in favour of this. It is at this time that a considerable gradient forms in the distribution of the velocities in the interior of the star which leads to the appearance of the emission lines of hydrogen. Afterwards the perturbation passes into the higher layers of the photosphere. The wave which it propagates rises from the interior of the star and moves out to the periphery.

Following this, we analysed the slow variations of brightness and radial velocity. As is known we are fully informed on the slow variations of the radial velocity only in the case of $U$ Monocerotis, thanks to the researches of R. Sanford. We have succeeded in discovering, by a harmonic analysis of the observations of A. Joy, the slow variation of the radial velocity in R Sagittae and RV Tauri, and to suspect the existence of similar variations in DF Cygni and SS Geminorum. We have studied also the slow variations of brightness that are synchronized with the variations of the radial velocity. As a result, we have obtained some ideas concerning the slow variations of stars of the RV Tauri type. The results can be summarized as follows:

(I) The curves of brightness and of radial velocity are in phase, if the negative values of the velocities are taken in the positive sense on the ordinate axis. Therefore, one cannot suppose that the slow variations of the velocity are caused by orbital motion; it follows that the slow variations of the radial velocities and of light are due to pulsation.

(2) The curves of the radial velocities yield, after their integration, the curves of variation of the radius of the reversing layer. It appears that in RV Tauri the radius varies by 380 million kilometres, in $R$ Sagittae by 374 million, and in U Monocerotis by 1250 million kilometres.

(3) Such large variations of the radius of the reversing layer are possible only in case the radius attains a value of several thousand million kilometres. It follows that the stars of the RV Tauri type are the largest of all those which we know.

The structure of a star of the RV Tauri type can be represented as follows: in the interior there exists a nucleus that possesses a photosphere extending to a radius nearly a hundred times greater than that of the Sun. This nucleus is surrounded by an enormous upper layer, which is an 
extended reversing layer having an outer radius of several thousand million kilometres.

A mechanical pulsation probably could not be the cause of the variations described above; it is possible that these variations have for an origin the variations of the effective gravity, which could be considerable due to the changes of the radial pressure. It is possible that this mechanism can explain the phase relationship between the light curves and the radial velocity curves of the cepheids. It is possible that the radial velocities of the cepheids vary as a result of the variations of the radial pressure in an extended reversing layer, where the absorption lines are formed.

The question thus raised in the study of the RV Tauri stars makes it possible to re-examine from another viewpoint the properties of the cepheids.

\section{REFERENGES}

[1] F. Loukatskaya, Variable Stars, 9, 57 (1952).

[2] A. M. Shulberg, Variable Stars, 9, 256 (1953); Publ. Astr. Obs. Univ. Odessa, 3, 249 (1953).

[3] I. G. Jdanova and V. P. Tsesevich, Publ. Astr. Obs. Univ. Odessa, 3, 3 (1953). 